\title{
Práctica pedagógica para la enseñanza de la gimnasia artística: Etapa de iniciación deportiva
}

\section{Pedagogical practice for teaching artistic gymnastics: Sports initiation stage}

\author{
http://dx.doi.org/10.17981/cultedusoc.12.2.2021.06
}

Recibido: 14 de junio de 2020 Aceptado: 21 de octubre 2020 Publicado: 1 de julio de 2021.

\author{
Heriberto González-Valencia \\ Institución Universitaria Escuela Nacional del Deporte. Cali (Colombia) \\ hery77@hotmail.com \\ German Darío Isaza-Gómez (1) \\ Institución Universitaria Escuela Nacional del Deporte. Cali (Colombia) \\ german.isaza@endeporte.edu.co \\ Luz Karime Parra-Estrada (1) \\ Institución Universitaria Escuela Nacional del Deporte. Cali (Colombia) \\ luz.parra@endeporte.edu.co
}

Para citar este artículo:

González-Valencia, H. Isaza-Gómez, G. y Parra-Estrada, L. (2021). Práctica pedagógica para la enseñanza de la gimnasia artística: Etapa de iniciación deportiva. Cultura, Educación y Sociedad, 12(2), 95-112. DOI: http://dx.doi.org/10.17981/cultedusoc.12.2.2021.06

\section{Resumen}

Este artículo pretende contribuir a fomentar la conciencia de deportistas y entrenadores acerca de la importancia de prevenir cualquier tipo de lesión en deportistas en iniciación. El objetivo es analizar la practica pedagógica para la enseñanza de la gimnasia artística en etapa de iniciación deportiva de un club de la ciudad de Cali (Colombia). La metodología se fundamenta en un paradigma mixto que se enfoca en comprender la interpretación y medición de los resultados, desde la integración de la información obtenida a través de técnicas como observación y encuestas para los datos cuantitativos y las entrevistas y revisión documental en el componente cualitativo. Se trabajó con 10 entrenadores de un club de enseñanza de gimnasia artística en la ciudad de Cali (Colombia) en el período 2020-A. Los resultados evidencian que la gimnasia por ser un deporte bastante complejo y a su vez de un alto grado de esteticidad requiere una preparación física general y específica sobre cada aparato independientemente de los contenidos a enseñar. Se concluye en la importancia de analizar y validar la practica pedagógica para la enseñanza de la gimnasia artística en etapa de iniciación deportiva en el club.

Palabras clave: Gimnasia artística; iniciación deportiva; prácticas pedagógicas

\section{Abstract}

This article aims to contribute to promoting the awareness of athletes and coaches about the importance of preventing any type of injury in athletes in initiation. The objective is to analyze the pedagogical practice for the teaching of artistic gymnastics in the sports initiation stage of a club in the city of Cali (Colombia). The methodology is based on a mixed paradigm that focuses on understanding the interpretation and measurement of the results, from the integration of the information obtained through techniques such as observation and surveys for quantitative data and interviews and documentary review in the qualitative component. We worked with 10 coaches from an artistic gymnastics teaching club in the city of Cali (Colombia) in the 2020-A period. The results show that gymnastics, because it is a fairly complex sport and, in turn, has a high degree of aesthetics, requires general and specific physical preparation on each apparatus regardless of the content to be taught. It concludes on the importance of analyzing and validating the pedagogical practice for the teaching of artistic gymnastics in the sports initiation stage in the club.

Keywords: Artistic gymnastics; sports initiation; pedagogical practices 


\section{INTRODUCCIÓN}

La orientación principal del artículo es analizar la práctica pedagógica para la enseñanza de la gimnasia artística en etapa de iniciación deportiva, ya que muchos entrenadores, desde su punto de vista empírico establecen distintos modelos de cómo se debe enseñar la gimnasia, dificultándose no solo para el deportista, sino también en relación con las metas deportivas que se ha planteado el entrenador. González, García, Contreras y Sánchez-Mora (2009) proponen la iniciación deportiva como un momento comprendido en edades de 6 a 15 años aproximadamente, teniendo en cuenta modificaciones acorde al desarrollo individual de la experiencia previa del deportista, también dan por hecho que en cualquier edad se puede iniciar el deporte. Por otro lado, González, Villota y Riofrio (2019) argumentaron que los profesores están permeados del método tradicional, lo que puede llevar a una barrera en el avance de nuevos métodos. González (2015) menciona que los educadores deben estar al día con las tendencias en la educación y así poder desarrollar diferentes actividades en sus procesos.

La gimnasia artística es un deporte donde los gimnastas presentan altos riesgos al momento de ejecutar los ejercicios, por lo cual es notorio la falta de los procesos de enseñanza en la iniciación deportiva, donde se pueda fijar el cuidado de los deportistas, la metodología de enseñanza, los medios que se utilizan para la realización de dichos ejercicios, las estrategias pedagógicas de enseñanza ya que una gran parte de los entrenadores basan sus entrenamientos enseñando la gimnasia como un deporte profesional antes de enseñarla como la iniciación de ella; es por eso que la falta de los procesos de enseñanza han llevado al deporte (la gimnasia artística) en algunos casos tanto nacionales como internacionales a múltiples accidentes aparatosos que no pueden ser remediados; como lo fue el caso de la soviética Elena Mujina, hace 35 años, quien debido a la presión por parte de su entrenador en los entrenamientos previos a competencias sufrió una lesión la cual la dejo tetrapléjica.

Se ha evidenciado que los accidentes no se dan solo por la inadecuada elaboración de los ejercicios de alta dificultad en la gimnasia, sino también por el escaso perfil pedagógico en los entrenadores para el cuidado de la enseñanza de la gimnasia artística desde la etapa de la iniciación deportiva, ya que gran parte de entrenadores, no solo en la gimnasia si no en el deporte en general, realizan sus prácticas desde lo empírico limitándose solo en su experiencia como deportista en el deporte que han practicado y no procesos formativos orientados a la apropiación de una didáctica deportiva. Araujo (2004) resaltó que la integridad física y psíquica de los niños y jóvenes nos preocupa realmente, pero también tenemos muy claro que una ejecución correcta es el mejor camino para evitar accidentes y lesiones. Los ejercicios deben incorporar diversos aspectos propios de este deporte en el que se traten de propiciar las condiciones físicas y psíquicas necesarias para su vida deportiva (Capote, Rodríguez, Analuisa, Vivas y Alomoto, 2017).

El cuidado en la gimnasia se basa fundamentalmente en el seguimiento lo más cercano posible de los gimnastas, en estar atentos a cada movimiento y expresión; teniendo en cuenta la escasa literatura científica relacionada con el cuidado de la gimnasia en general, 
desde un punto de vista clínico, Ceballos (2010) señala desde la enfermería que el cuidado humanizado es más que un buen trato o satisfacción usuaria, es que "el otro» necesita ser cuidado y al realizarlo el usuario obtiene beneficios, no sólo el paciente sino también el profesional y el equipo que desarrolla los cuidados. Capote et al. (2017) mencionan que los ejercicios y sus particularidades son de necesario conocimiento por parte del entrenador deportivo para poder estimular correctamente a sus estudiantes, por tal razón es necesario caracterizar los mismos teniendo en cuenta las particularidades del deporte, edad del niño, así como sus características individuales.

Por esta razón este artículo se centra en los entrenadores de gimnasia de la ciudad de Cali (Colombia), donde se evidencian dificultades para la enseñanza de la gimnasia artística en la etapa de iniciación deportiva, también se pretenden develar las debilidades que pueden tener los entrenadores en sus enseñanzas desde sus percepciones, sus niveles de preparación, y de esta manera proveer visiones amplias en la pedagogía y métodos de enseñanza, donde es decisión de cada entrenador modificar sus metodologías para las gimnastas, dependiendo de lo que se considere viable.

Es importante los aportes a la práctica pedagógica del deporte que también se podrá ampliar en diferentes ámbitos ya que la pedagogía puede ser considerada por muchos alumnos y profesores como una de las disciplinas académicas más importantes, porque conlleva a la enseñanza y aprendizaje de los diferentes actores. Díaz (2006) destaca que la formación docente puede ser examinada a través de dos importantes categorías de análisis: la práctica pedagógica y el saber pedagógico, las cuales se caracterizan por su complejidad. También se infiere impacto en función del análisis de la practica pedagógica y su aplicación en clases por medio de la labor didáctica de los entrenadores y la intervención activa de las gimnastas en el proceso.

\section{FUnDAMENTACIÓN TEÓRICA}

\section{Gimnasia artística. Conceptualizaciones básicas}

La Federación Colombiana de Gimnasia (Fedecolgim, 2021) define este campo como una disciplina deportiva donde ser realizan composiciones coreográficas, y combinan en forma simultánea y a una gran velocidad, los movimientos del cuerpo, lo que exige deportistas con condiciones físicas excepcionales. Este es un deporte olímpico donde las mujeres y hombres compiten en diferentes aparatos por separado, se puede afirmar que esta es una de las disciplinas modernas más antiguas desde el comienzo de las olimpiadas. Históricamente, la gimnasia artística se ha desarrollado dentro de la transformación progresiva de una figuración aficionada a otra profesional (Sánchez y Martín, 2019).

Tous (2002) menciona que en la gimnasia; la técnica y didáctica en el desarrollo de la actividad gimnastica y su investigación científica ha sido procedido de largos periodos donde la práctica antecedía al conocimiento teórico. La integración de la gimnasia artística dentro del movimiento deportivo internacional y el desarrollo del formato tal y como la conocemos en la actualidad fue algo progresivo (Sánchez y Martín, 2019). Santos (2020) argumenta que la Gimnasia Artística es un deporte con larga historia a nivel 
internacional y con una marcada presencia en actual, principalmente en los espacios de enseñanza. Esto revela una tendencia natural de nuevas generaciones interesadas en incursionar en esta rama deportiva.

Araujo (2004) aduce también que el aprendizaje de las técnicas y la seguridad de los deportistas, y la integridad física y psíquica de los participantes es un factor que se tiene en cuenta, pero también que una correcta ejecución es la forma ideal para prevenir accidentes y lesiones. Factores muy relevantes para establecer practicas pedagógicas que aseguren la integridad de los/las practicantes de la gimnasia artística, especialmente cuando están en procesos de inicio.

\section{Estrategias pedagógicas en la enseñanza de la gimnasia artística}

Valencia, Enríquez y Agredo (2017) definen estrategias pedagógicas como aquellas orientaciones que el profesor da a estudiantes para promover el aprendizaje. Fraile (2004) alega que los entrenadores de actividad física y deportiva deben tener la principal responsabilidad de evidenciar las finalidades de las practicas físico-descriptivas que se vienen ofreciendo en edad escolar; cuáles son sus contenidos de enseñanza; como se llevan a cabo; etc. Este autor también menciono que se debe tener una postura de compromiso hacia los valores que sean coherentes con las tareas educativas de la escuela (Fraile 2004). Se debe pretender en todo momento poder visualizar estrategias que lleven a obtener logros, sin dejar a un lado todas las prevenciones de cualquier posible lesión.

Giglio (2013) propone que la colaboración creativa modifica la forma de enseñar, creando una propuesta pedagógica de colaboración creativa y reflexiva y luego continuar con la observación para así comprender que es lo que sucede cuando se aplica tal pedagogía en lugares distintos con personas diferentes, trabajando juntos los estudiantes con los docentes para componer ellos mismos lo que buscan en la investigación. Giglio (2013) además observó los diferentes enfoques educativos que actúan sobre la improvisación en la enseñanza escolar ya que son limitados y difíciles de ser enseñados de manera tradicional teniendo, así como un objetivo principal donde el docente tenga un papel de importancia en el sostenimiento de la orientación de la actividad planificada por el mismo. Es por eso, que la innovación a través de buenas prácticas pedagógicas puede hacer la diferencia en los procesos de enseñanza.

Aval (2016) explica que una propuesta innovadora puede derivar de un paradigma pedagógico donde se visualice un marco teórico en construcción y un modo de intervención organizado y secuenciado en etapas. Fernández-Río, Calderón, Alcalá, Pérez-Pueyo y Cebamanos (2016) exponen modelos pedagógicos en educación física, donde la comprensión se hace necesaria para el desarrollo de un proceso educativo de calidad. Gómez (2005) plantea estilos, estrategias y técnicas de enseñanza más propicias para incrementar la autonomía. Como por ejemplo las estrategias emancipativas, los estilos de enseñanza de investigación y técnicas de enseñanza como trabajos de forma semidefinida (en la que se le plantea al estudiante el objetivo de la asignación motriz a realizar, pero no así la forma de ejecutarla). 
Toro, Álvarez y Benjumea (2013) argumentan que desde las prácticas del campo de la educación física se pueden superar las tendencias tradicionales de técnica, disciplina, de regularización; y deben ser convertidos en momentos creativos, lúdicos, generadores de posibilidades de verdaderas experiencias de inclusión. La actualidad de la educación permite poner en practica estos argumentos, los entrenadores cuentan con variedad de herramientas académicas para ofertar novedosas formas de enseñanza y practica deportiva, haciendo el proceso mucho más ameno y significativo.

\section{Procesos de enseñanza en iniciación deportiva}

Giménez, Abad y Robles (2010) mencionan la necesidad de entender la iniciación deportiva desde dos perspectivas diferentes. Por un lado, esta etapa forma parte del proceso de formación a largo plazo del deportista, pero a la vez, debe tener suficiente importancia por sí misma. La iniciación deportiva puede ser el punto de decisión de un deportista en encaminar su vida; se convierte en el momento de obtener unas bases solidas para el buen desempeño a través del tiempo.

Las estrategias utilizadas por profesoras en formación proponen una enseñanza donde se definen como aquellas orientaciones que el profesor utiliza para el desarrollo de una situación de enseñanza propuesta por el mismo, comparte o proporciona una determinada situación de enseñanza al estudiante donde a través del desarrollo implementando distintas estrategias de enseñanza (González, Ibáñez, Feu y Galatti, 2017).

Castejón (2015) propone cambios significativos en la iniciación deportiva con el fin que el deporte tenga sentido educativo al incidir en los ámbitos cognitivo, afectivo social y motor. Por otro lado, Gómez-Mármol, Calderón-Luquin y Valero-Valenzuela (2014) utilizaron el modelo de enseñanza para la iniciación ludo técnico donde las tareas de aprendizaje utilizadas se apartan de lo tradicional, y se diversifican en función de la parte de la sesión, destacando de entre ellas, las denominadas propuestas ludo técnicas. Estas propuestas pueden hacer la diferencia en cuanto unas bases sólidas de aprendizaje, y a su vez, una motivación de los deportistas en su continuidad.

Rodríguez, Lara y Rodríguez (2018) reflexionaron acerca de la iniciación deportiva en cuba y específicamente al entrenador encargado de esta, y establecieron la necesidad de un modelo de desempeño hacia el educador donde se diferencien las funciones de las del entrenador deportivo y de las del profesor de educación física para obtener como conclusión, la iniciación deportiva como proceso.

\section{Métodos de enseñanza deportiva}

Ortiz y Canto (2013) proponen que desde el punto de vista de la didáctica se construye la vía más importante para la dirección del proceso de enseñanza y se usa para el desarrollo de hábitos y habilidades, la impartición de conocimientos y también ayudan a que las nuevas generaciones logren asimilar la experiencia social. Díaz, Martínez y Vernetta (2004) evidenciaron la división de los procesos académicos y los dividieron a nivel descriptivo, a nivel de correlacional y a nivel experimental donde se evidencian los cambios. 
Castejón y Giménez (2003) argumentan que los cambios permanentes, sobre todo en la didáctica y en la psicología del aprendizaje tienen un efecto en la forma de asimilar la enseñanza y el aprendizaje del deporte en la escuela. Davini (2008) menciona que el método de enseñanza necesita de principios y criterios básicos de intervención, que posibiliten la consecución metódica de sus fines con los medios más adecuados.

Hernández (2015) propone una estrategia metodológica que mejore los procesos en la educación, permitiendo innovar en los procesos educativos, potenciando enormemente la formación y aprendizaje. Arellano, Fernández, Rosas y Zúñiga (2014) consideran significativo innovar en las propuestas de enseñanza con la integración de recursos tecnológicos desarrollando capacidades y competencias necesarias en los estudiantes en iniciación. Es una realidad que con los cambios tecnológicos que ha vivido la humanidad entera, la educación debe tener reformas con la integración permanente de procesos innovadores y significativos, donde los más beneficiados sean los aprendices.

Guillen, Copello, Gutiérrez y Guerra (2018) proponen que hay estrategias pedagógicas a partir de talleres de superación, contexto en el que se garantizaron los conocimientos necesarios en el orden teórico y metodológico para apreciar el proceso desde una nueva perspectiva que permitiera solventar las limitaciones constatadas en los procesos académicos. Pires y Yanes (2013) destacaron el planteamiento docente a través del desarrollo de su innovación. Por otro lado, Fernández-Río (2013) diseñó un marco metodológico para la enseñanza de las habilidades gimnásticas en un contexto educativo, hablando de las habilidades gimnásticas que constituyen un contenido curricular básico en la educación física.

\section{Metodología}

Este artículo de investigación de enfoque mixto, cualitativo y cuantitativo, aborda un análisis de las categorías estudiadas. Según Otero (2018), la integración de ambos componentes en un mismo diseño contribuye a la representación de un proceso sistemático, empírico y crítico de la investigación para dar respuesta a problemas humanos. Cauas (2015) argumenta que este tipo de investigaciones se enfocan en la utilización práctica de los resultados de un estudio con base en una rigurosa descripción contextual de los hechos o situaciones. Otero (2018) argumenta que el enfoque mixto se caracteriza porque favorece una perspectiva amplia y profunda del fenómeno que se estudia, así mismo, esto le permite al investigador plantear el problema con claridad y formular objetivos.

La investigación es descriptiva, ya que permitió establecer las formas utilizadas por los entrenadores de gimnasia con respecto a las deportistas para realizar clases de gimnasia con diferentes grupos en la etapa de iniciación deportiva y analizar cómo es su enseñanza. Se aplicaron encuestas y entrevistas, donde se recogieron datos relevantes de análisis. Vargas (2020) argumenta que la entrevista permite la profundización de los investigadores en ciencias sociales y es uno de los métodos cualitativos empleados en la investigación porque posibilita que el entrevistado sea franco en sus comentarios. Schettini y Cortazzo (citados por Vargas, 2020) precisan que la entrevista se caracteriza por ser un proceso 
comunicativo que se da en un encuentro entre sujetos, donde la finalidad es acceder a la perspectiva de los sujetos.

Por otro lado, Hernández y Velasco-Mondragón (2000) mencionan que la encuesta debe considerar aspectos relacionados con la población en estudio, los individuos de quienes se obtiene la información y la información que se busca obtener. López (2004) describe la población como el conjunto de individuos u objetos de los que se desea conocer algo en una investigación y la muestra como una población en que se llevará a cabo la investigación. La muestra es una parte representativa de la población.

Se trabajó con (10) diez entrenadores de gimnasia quienes están en diferentes clubes deportivos y entrenadores de algunas instituciones educativas. Se hizo la selección de acuerdo con el nivel y edades que manejan en sus clubs, teniendo en cuenta su homogeneidad en el área de estudio. Arias-Gómez, Villasís-Keever y Miranda-Novales (2016) aseguran que:

La integración del grupo de sujetos o participantes de los estudios, con las características particulares que permitirán responder los objetivos planteados, es una parte fundamental de todo protocolo de investigación porque cuando se logra una apropiada selección, no solo se podrá disponer de resultados confiables, sino que es posible que dichos resultados puedan ser extrapolados a otras poblaciones similares. Además, una buena elección de los participantes para el proyecto cumple con el propósito esencial de asegurar que los hallazgos representarán, de forma exacta, lo que sucede en la población de interés.

\section{Técnicas e instrumentos}

Martínez y Galán (2014) mencionan que las técnicas se implementan para recoger información de la población objeto de un estudio el cual puede ser encuestas y entrevistas; por su parte el cuestionario es un instrumento muy útil para la recogida de datos de los sujetos a los que interesa considerar, permite, además, en paralelismo con la entrevista, identificar y sugerir hipótesis y validar otros métodos. Se realizó la encuesta como técnica para recolectar datos, ya que permiten tener una visión general por medio de estadísticas, se aplicó por medio de un cuestionario en línea.

Con la entrevista a cada entrenador, se obtuvo información concreta acerca de la practica pedagógica en la enseñanza de la gimnasia, lo cual permitió conocer el proceso como entrenadores, elaborándose por medio de las plataformas virtual Zoom y Meet.

También se tuvo en cuenta en la revisión documental, la cual Morales (2003) define como un procedimiento científico, un proceso sistemático de indagación, recolección, organización, análisis e interpretación de información, textos, documentos o datos en torno a un determinado tema, el cual permitió aclarar y resolver los problemas generados en esta investigación. Para esta investigación se hizo un rastreo exhaustivo de los antecedentes y la producción bibliográfica en torno al tema tratado.

Siendo también, por medio de la observación detallada de las clases de los entrenadores del club en el momento de la práctica, evidenciando si la utilización de los medios didácticos está presente para la etapa de iniciación deportiva, así como las principales dificultades del proceso. 


\section{Resultados}

Como resultado de este estudio se analizaron las entrevistas y encuestas realizadas a los diferentes entrenadores de gimnasia, por lo tanto, la investigación permitió conocer las características de la enseñanza de la gimnasia frente al grupo de deportistas que maneja cada uno, tanto de niveles pequeños como en los niveles grandes. Se obtuvo una línea similar en la enseñanza sin importar el tipo de nivel con el que se encuentren trabajando, al adquirir como resultados que la gimnasia por ser un deporte bastante complejo y a su vez de un alto grado de esteticidad es uno de los deportes más complejos del cual se requiere una preparación física general y específica sobre cada aparato sin importar lo que se esté enseñando.

Las características generales de la enseñanza conllevan un mismo punto para que el trabajo realizado en las clases den un resultado, el cual consiste en; trabajar con la variabilidad, la utilización de diferentes métodos de juego, la utilización del método deductivo, la división por fases para la enseñanza de los elementos desde lo más complejo a lo más difícil, claramente dependiendo de los grupos que se tengan así mismo se trabaja pero sin lugar a duda uno de los puntos clave para poder realizar una buena enseñanza en la gimnasia es la imaginación, lo que se debe tener presente ya que los deportistas deben realizar preparación física y, en este caso realizarlo por medio de los juegos para que no haya un aburrimiento o deserción del deporte. Este método ayuda a que las deportistas se motiven en las clases, y que no vean como una obligación si no como un gusto por ellas y con base a esas estrategias utilizadas en cada clase lograr resultados positivos en los entrenamientos.

Con relación al análisis de las entrevistas se encuentra similitud con el tema de la variabilidad frente a los entrenamientos, ya que la gimnasia presenta varias modalidades y dentro de ella presenta diferentes aparatos, no se puede obtener la concentración de un deportista en un solo aparato y se necesita de los demás, para conseguir los objetivos propuestos en la clase planificada por el entrenador necesitara de un arduo trabajo físico, pero para esto se llega a la variabilidad de los ejercicios en cuanto a la preparación física y a lo especifico ya que las clases según los entrenadores no pueden ser iguales a la del día anterior o incluso de la semana pasada, es ahí cuando se juega el papel de la imaginación y la utilización de los medios que se encuentren dentro de la sala o incluso también está la de salir del aérea de entrenamiento pero con sus respectivos cuidados. Todo esto hace percibir que el resultado final puede depender solo de la practica pedagógica de cada entrenador individualmente sin un seguimiento de lineamientos generales.

Por otro lado, se analiza el juego como practica pedagógica, el cual predomina en las edades tempranas para la aceptación de dichos ejercicios, para lograr hacer amenas las clases, atraer la atención de los deportistas más pequeños. Los entrenadores especializados en estas etapas recomiendan la utilización del juego casi que un $90 \%$ de las clases tanto los juegos individuales como los juegos cooperativos. Las dinámicas de juegos con identificación de nombres técnicos de elementos y posiciones de la gimnasia es un factor importante para la corrección de estos y su aprendizaje, según en esta parte para poder realizar dichos juegos o actividades lúdicas requiere de elementos lúdicos para lograr se llevada a cabo. 


\section{Particularidades metodológicas en los programas de enseñanza de la gimnasia en la ciudad de Cali, Colombia}

Para cada edad existen diferentes metodologías de enseñanza en la gimnasia, aun estando en la etapa de iniciación deportiva; una de las metodologías más importantes para trabajar una buena y correcta enseñanza de la gimnasia tiene que ver con la cantidad de niños que entrene en una clase, por cuanto este deporte requiere de la máxima atención de los grupos de entrenamiento sin importar las edades. Es importante no exceder el límite de 8 a 10 deportistas por entrenador (Figura 1).

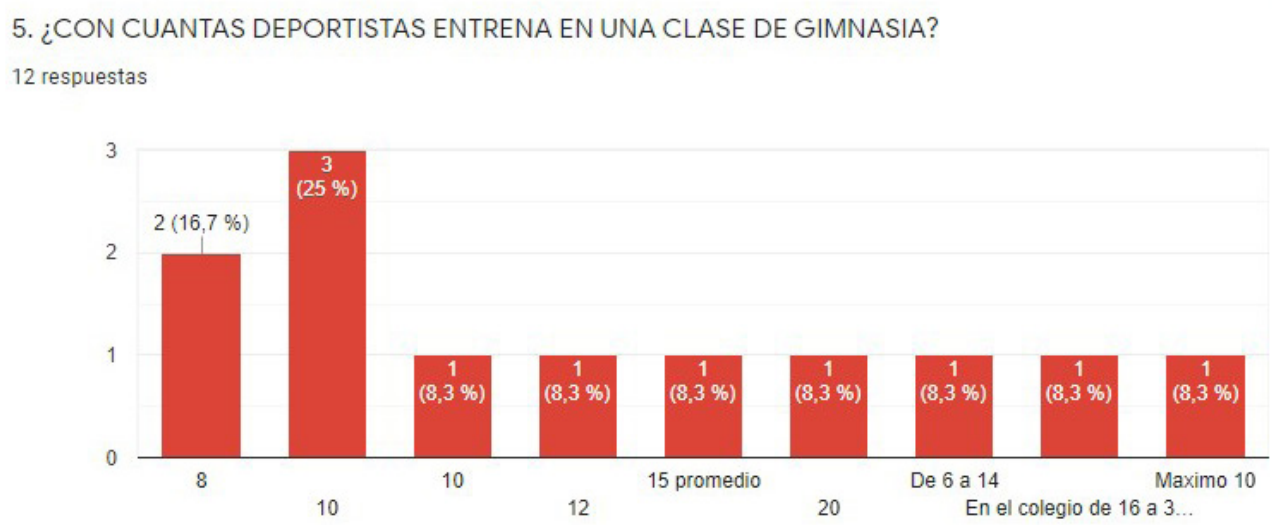

Figura 1. Número de deportistas en una clase de gimnasia. Fuente: Elaboración propia.

Según la Figura 1 se puede inferir que los clubes de gimnasia cumplen un protocolo más adecuado para la enseñanza en cuanto el manejo de las clases teniendo un máximo de 10 niñas en una clase de gimnasia, mientras que en los colegios el límite de niñas excede de 15 a 20 deportistas con tan solo 2 profesores, lo que puede percibirse como inseguridad ya que cada niña requiere un acompañamiento en los aparatos gimnásticos. Aunque en general el numero de deportistas es alto, ya que la iniciación en la gimnasia artística requiere de una alta concentración y un manejo casi que individual para la adquisición de buenas técnicas de ejecución.

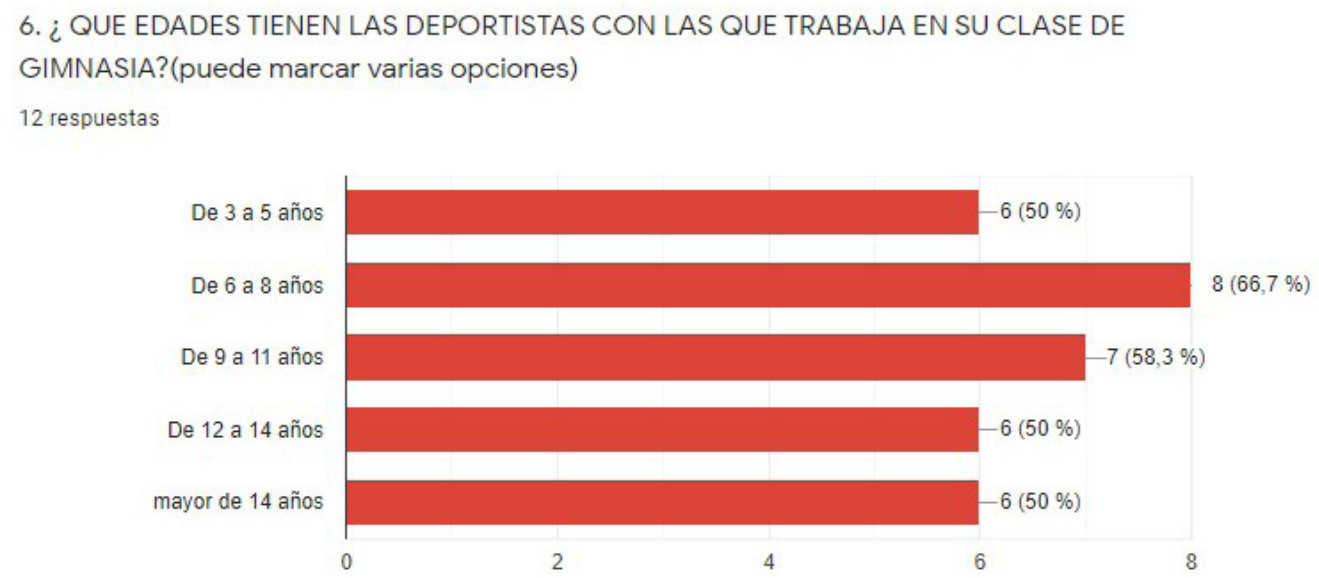

Figura 2. Edades de las deportistas con las que trabajan en los entrenamientos. Fuente: Elaboración propia. 
La siguiente Figura 2 muestra las edades que manejan 10 (diez) entrenadores de gimnasia de diferentes clubes y colegios de la ciudad de Cali.

Las edades de las deportistas van asociadas al desarrollo de la clase y en el nivel de aprendizaje; los entrenadores tienen algunas similitudes de métodos teniendo en cuenta que casi todos trabajan con las mismas edades, por muy alto o bajo nivel que tengan al tratarse de niñas en etapas de desarrollo y crecimiento necesitan vivenciar esta etapa de la gimnasia, como un espacio donde todo su cuerpo cambiara físicamente en vista de los ejercicios tan complejos que esta disciplina requiere; para poder llevar a cabo toda la actividad física también es de vital importancia dicha preparación, el trabajo de estos entrenadores comienza cuando cambian la preparación física a métodos de juegos para no perder la concentración y la diversión al mismo tiempo ya que siguen siendo niñas deportistas. Todo esto evidencia como una buena practica pedagógica puede hacer la diferencia en los procesos de iniciación de las deportistas.
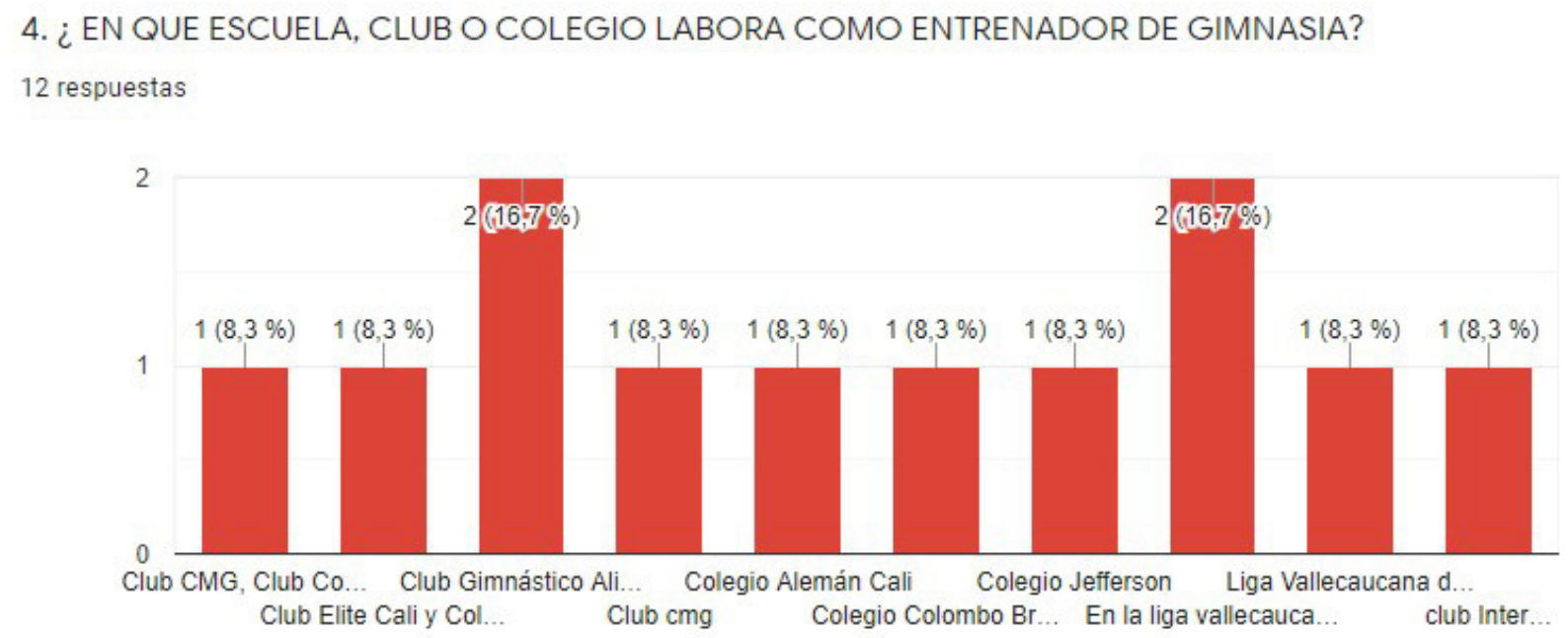

Figura 3. Lugar donde realizan la clase de gimnasia.

Fuente: Elaboración propia.

La Figura 3 evidenció que los 10 (diez) entrenadores encuestados manejan grupos de diferentes edades, por cuanto los entrenadores que cumplen labores en los colegios y clubes al mismo tiempo son personas con perfiles más acordes al trabajo con diferentes tipos de edades; más específicamente los entrenadores que pueden trabajar con las edades tempranas son los más capacitados para trabajar con edades mayores, en cambio los entrenadores que manejan grupos de edades grandes, presentan algunas dificultades con los grupos pequeños, esto se puede evidenciar según el diario de campo y la forma de manejo de grupos. Esto muestra como algunos procesos se pueden ver afectados por la capacidad individual de cada entrenador.

La encuesta aplicada mostró que todos los entrenadores son personas capacitadas para realizar una clase de gimnasia con un mínimo de estudio; el pregrado y un porcentaje de ellos con un estudio de posgrado, de acuerdo con la indagación bibliográfica, conlleva a una mejor practica pedagógica en los procesos de enseñanza y aprendizaje. 
3. ¿CUAL ES SU NIVEL DE ESTUDIO MAS ALTO?

12 respuestas

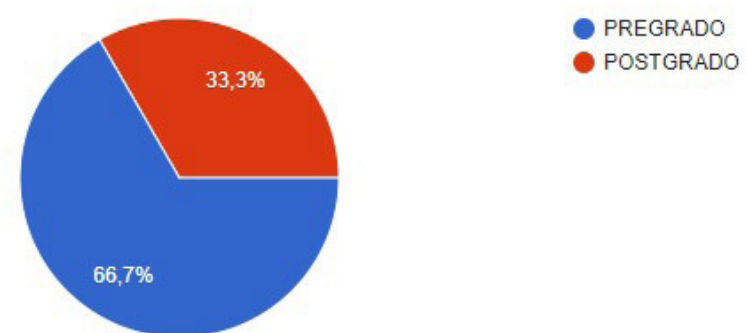

Figura 4. Nivel de estudio de los entrenadores.

Fuente: Elaboración propia.

Los 10 (diez) entrenadores cuentan con las exigencias mínimas para un entrenador de gimnasia, las cuales son las actualizaciones y seminarios realizados en distintas etapas del año en la ciudad y/o fuera de la ciudad, por lo tanto, todos lo entradores utilizan la pedagogía como un método de enseñanza y a su vez la describen como una parte predominante en la enseñanza no solo de la gimnasia si no de cualquier actividad o clase que se dé a niños.

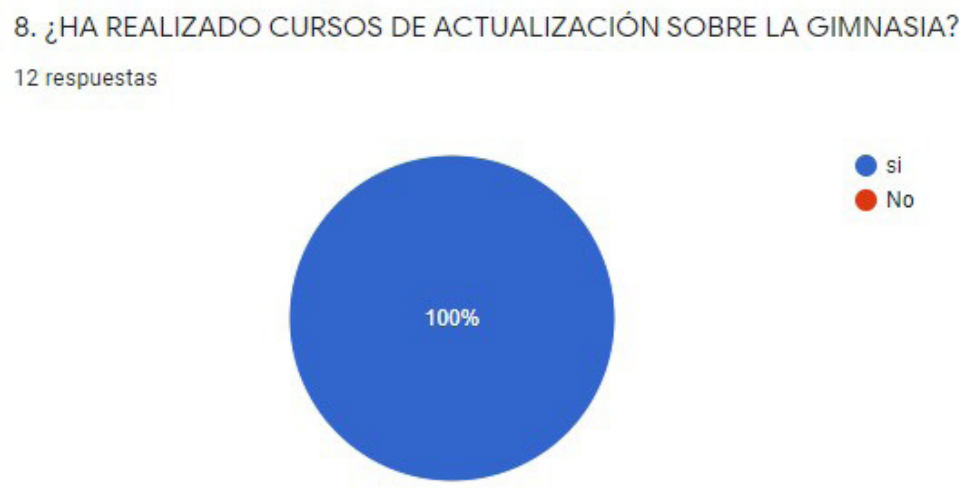

Figura 5. Realización de cursos de actualización en la gimnasia.

Fuente: Elaboración propia.

Los siguientes gráficos muestran el 100\% de la aceptación de los entrenadores como respuesta a los seminarios, actualizaciones, al uso y la importancia de la pedagogía en sus clases.

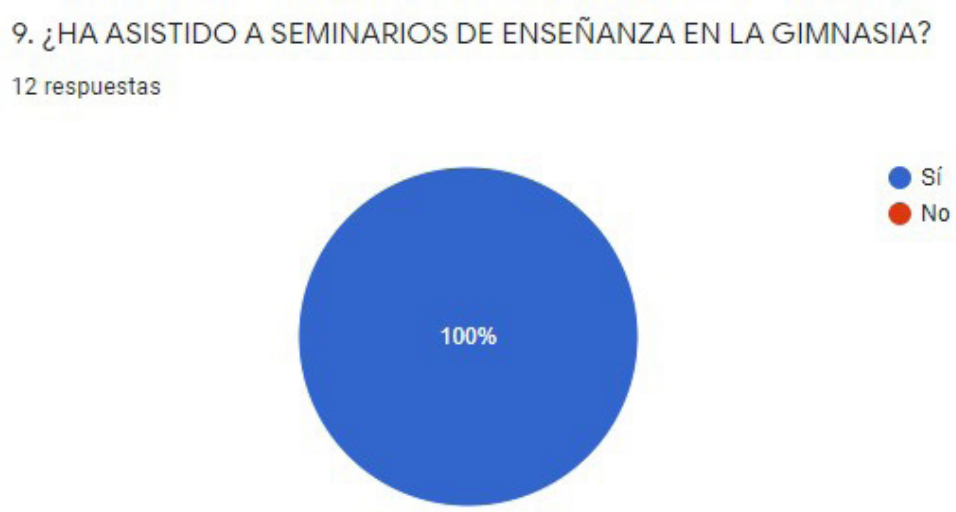

Figura 6. Asistencia a seminarios de la gimnasia.

Fuente: Elaboración propia. 
El 100\% de los entrenadores manifestaron la integración de prácticas pedagógicas en los procesos de iniciación en la gimnasia artística como medio significativo, buscando que las deportistas estén en un ambiente de aprendizaje agradable y con los resultados esperados al final del proceso.

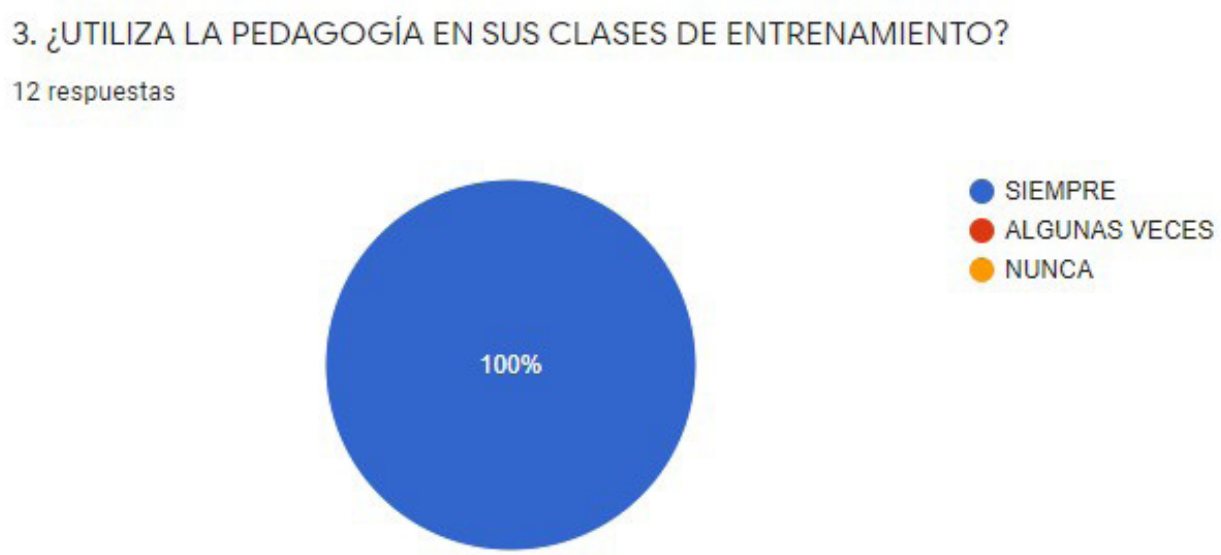

Figura 7. Utilización de pedagogía en los entrenamientos.

Fuente: Elaboración propia.

Con respecto a las metodologías utilizadas y contenidos abordados se evidencia la enseñanza de diferentes componentes de la gimnasia artística por partes, individualizándolos; también la utilizan por medio de la enseñanza directa y la visualización de cada uno de los elementos.

\section{2. ¿PARA USTED QUE TAN IMPORTANTES ES LA PEDAGOGIA EN EL ÁMBITO DEPORTIVO? \\ 12 respuestas}

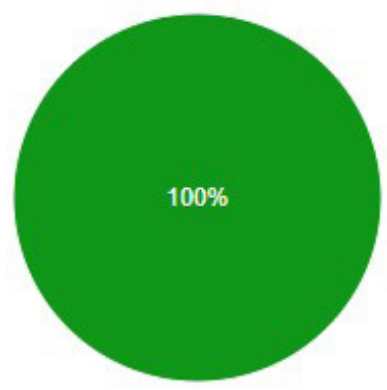

Figura 8. Importancia de la pedagogía en el ámbito deportivo. Fuente: Elaboración propia.

Las horas de entrenamiento son muy importantes, ya que en la etapa de iniciación deportiva se promueve que las niñas no deserten el deporte por entrenar de más y que se sientan aburridas, es por eso que las encuestas con los entrenadores arrojan datos importantes ya que la mitad de ellos utilizan más de dos horas de entrenamiento lo que en la iniciación deportiva no es recomendable por el beneficio y la salud de las deportistas. Tomando en cuenta que los entrenadores que trabajan más de dos horas son de procesos avanzados y que van direccionados hacia un proceso deportivo con mayor exigencia. De acuerdo con los antecedentes consultados, es recomendable comenzar con unas jornadas de entrenamiento muy flexible y sin tanta exigencia. 


\section{7. ¿CUANTAS HORAS DE ENTRENAMIENTO UTILIZA EN UNA CLASE DE GIMNASIA?}

12 respuestas

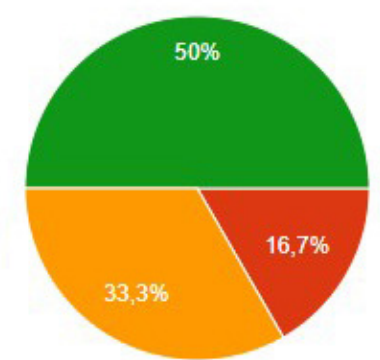

1 HORA

1 HORAY 30 MINUTOS

2 HORAS

- MAS DE 2 HORAS

Figura 9. Horas que utilizan en las clases de entrenamiento.

Fuente: Elaboración propia.

Otra de las particularidades de la enseñanza está relacionada con la vida deportiva de los entrenadores, aun cuando son personas estudiadas y actualizadas en el deporte, deben haber practicado la gimnasia, una de las ramas de la gimnasia o incluso otros deportes individuales o en conjunto que requieran de una máxima exigencia, así como la técnica y la esteticidad a la hora de practicarlo. Es necesario ubicarse en el lugar del deportista, el saber cómo piensa, si la forma de cuidarlos es cómoda para poder realizar ejercicios y hasta el punto de saber su límite en entrenamientos. A partir de lo expuesto se evidencia la importancia de los aportes de cada entrenador desde su propia experiencia como deportista, donde puede implementar y mejorar lo necesario para un optimo manejo de las deportistas en su etapa inicial.
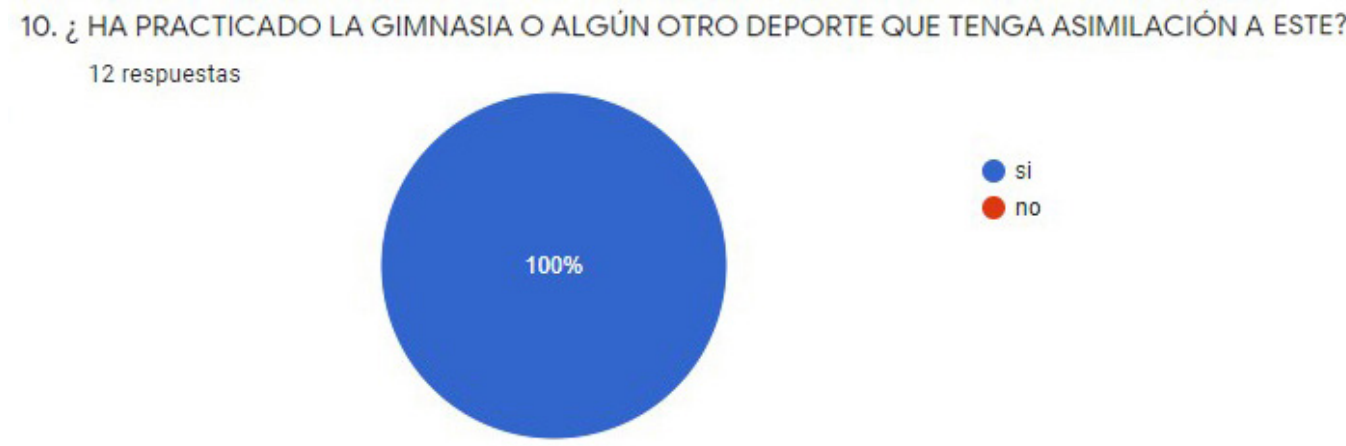

- no

Figura 10. Participación en la gimnasia u otro deporte como deportista. Fuente: Elaboración propia.

\section{SI SU RESPUESTA ANTERIOR FUE (SI). ESCRIBA CUAL.}

12 respuestas

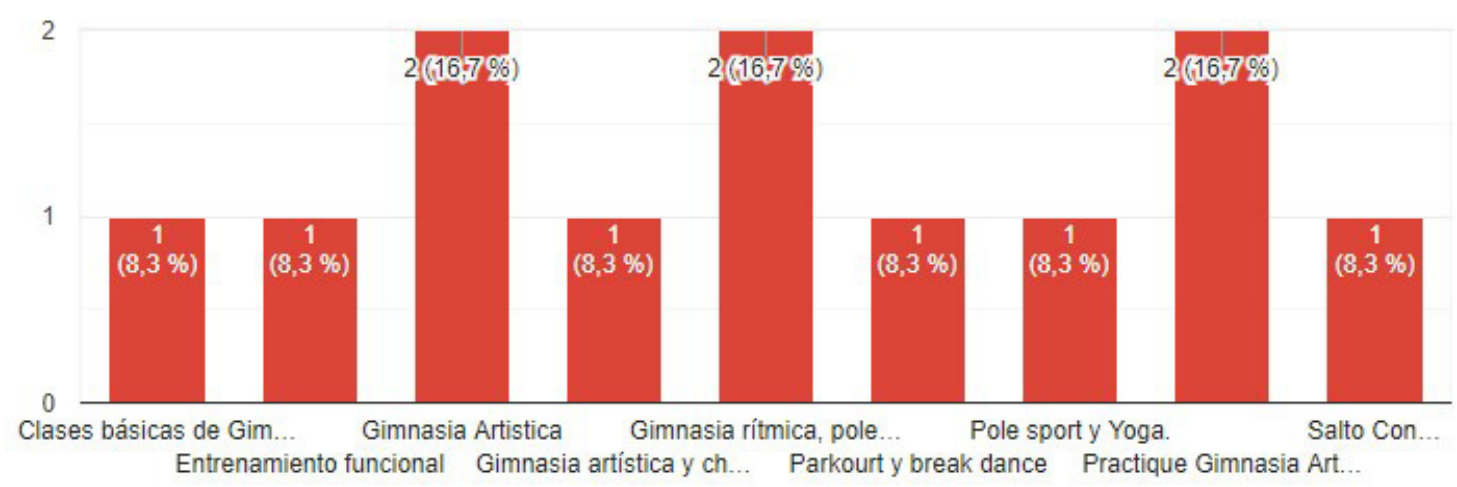

Figura 11. Deporte practicado.

Fuente: Elaboración propia. 
Como se puede observar en las gráficas anteriores, todos los entrenadores han practicado deportes similares a la gimnasia, como lo son el cheer leading, gimnasia artística, gimnasia rítmica, pole sport, parkourt y break dance, yoga y salto con garrocha, las cuales se puede concluir que al estar como entrenadores les favorece la enseñanza en cuanto a la explicación visual y verbal. Tener un recorrido y estar acostumbrados al lenguaje deportivo, amplía la gama de oportunidades de buen manejo de las deportistas.

\section{Conclusiones}

En primera instancia, se logró describir las características generales de la enseñanza de la gimnasia en los clubs e instituciones en las cuales se realizó la observación e intervención. Se detalló que los diferentes entrenadores manejan similitudes en los procesos de enseñanza en la gimnasia artística tanto en lo pedagógico como en el uso de los materiales didácticos, se tiene en cuenta que en la encuesta donde participaron 10 entrenadores y en la entrevista con una participación de 9 entrenadores describieron que trabajan por medio de juegos para el aprendizaje de los elementos o posturas gimnásticas. Donde todos los entrenadores toman la parte técnica de la gimnasia como la más importante en las sesiones de entrenamiento.

Los entrenadores de gimnasia, sin importar la especialidad, cuentan con formas similares de enseñanza teniendo en cuenta la necesidad de los deportistas, la enseñanza desde la parte del desglosamiento de los ejercicios hasta la parte de las correcciones ya que en todas las clases se tiene corregir hasta la parte más "fácil".

En segunda instancia, se identifican las particularidades metodológicas de los programas de enseñanza en la gimnasia en la ciudad de Cali, Colombia. Entre esas particularidades, los estudios realizados, sitios de trabajos en colegios y clubes, edades que trabajan, cursos de gimnasia y actualizaciones de estas, horas de entrenamiento por clases, y utilización de metodologías.

Se evidencio un análisis completo de las practicas pedagógicas en la enseñanza por parte de los entrenadores del club. Lo que hace un gran aporte a los entrenadores del club para lograr obtener un proceso de iniciación adecuado a las deportistas.

Se concluye que es oportuno realizar este tipo de investigaciones en el campo de la gimnasia y específicamente en el campo del deporte ya que como entrenadores o profesores, las mediaciones educativas, los procesos metodológicos, y medios que son utilizados en los entrenamientos son importantes para las etapas de formación de las niñas cuando entran al ámbito deportivo, esto le permitirá a los entrenadores identificar los problemas que tienen sus deportistas en el proceso dentro y fuera de la clase de entrenamiento y como entrenador podrá llevar a cabo soluciones para las deportistas.

Para finalizar, los profesores o entrenadores al efectuar este tipo de investigaciones son capaces de contribuir con una formación integral de los deportistas, aparte de trasformar sus clases de entrenamiento no solo en un gimnasio si no un espacio para ellos como distracción, ayuda psicológica y aprendizaje, el entrenador también llevara a cabo la labor de ser un amigo y confidente de los deportistas donde esto permitirá la confianza de los deportistas en lo que están realizando. 
Se recomienda a los entrenadores considerar unos lineamientos en la práctica pedagógica de la gimnasia artística, donde se destaca:

- Utilización adecuada de los medios didácticos para que las deportistas se sientan cuidadas y protegidas para realizar la práctica deportiva y superarse de manera fácil en los elementos acrobáticos.

- Programar clases donde se pueda implementar todo lo elaborado, tomando apuntes de los resultados de cada clase de entrenamiento, programando un tiempo determinado para que sea analizada cada clase y ver los pros y contras.

- Requerir el cumplimiento de los acuerdos de clases de entrenamiento a las deportistas al igual que como entrenador cumplir, para así llevar un entrenamiento agradable.

- Adaptar los métodos y técnicas de acuerdo con la iniciación deportiva pero aún más, a las edades con las que se esté trabajando, incluyendo todo lo elaborado en la propuesta como los métodos de juego, la explicación visual, verbal y por ayudas para lograr el entendimiento del ejercicio.

- Realizar clases en espacios fuera del club para que conozcan diferentes escenarios deportivos, teniendo en cuenta lo que se busca realizar fuera o en los diferentes sitios cuidando el bienestar del deportista.

- Hablarle en positivo a las deportistas, para que no sienta la palabra "prohibido" en sus entrenamientos si no buscar la manera de hacerles ver que tienen un riesgo y que es mejor esperar al entrenador.

- Por medio de los juegos, didácticas o diferentes enseñanzas aplicar siempre la gimnasia sin desviarse del tema o elemento que se está enseñando.

\section{REFERENCIAS}

Araujo, C. (2004). Manual de ayudas en gimnasia (Bicolor). Badalona: Paidotribo.

Arellano, N. M., Fernández, J., Rosas, M. V. y Zúñiga, M. E. (2014). Estrategia metodológica de la enseñanza de la programación para la permanencia de los alumnos de primer año de Ingeniería Electrónica. TE \& ET, (13), 55-60. Disponible en http://sedici.unlp. edu.ar/handle/10915/39993

Arias-Gómez, J., Villasís-Keever M. Á., Miranda-Novales, M. G. (2016). El protocolo de investigación III: la población de estudio. Revista Alergia México, 63(2), 201-206. https:// doi.org/10.29262/ram.v63i2.181

Aval, I. (2016). Aprendizaje servicio solidario: una propuesta pedagógica innovadora. RIDAS. Revista Iberoamericana de Aprendizaje Servicio, (2), 3-32. Disponible en https:// revistes.ub.edu/index.php/RIDAS/article/view/RIDAS2016.2.2

Capote, G., Rodríguez, Á., Analuiza, E., Vivas, Y. y Alomoto, M. (2017). La estimulación temprana en la gimnasia artística. Educación Física y Deportes, Revista Digital, 2(230). Disponible en https://www.efdeportes.com/efd230/la-estimulacion-tempranaen-la-gimnasia-artistica.htm 
Castejón, F. J. (2015). La investigación en iniciación deportiva válida para el profesorado de educación física en ejercicio. Retos, 28, 263-269 Disponible en http://hdl.handle. net/10486/678079

Castejón, F. y Giménez, F. (2003). Iniciación deportiva. La enseñanza y el aprendizaje comprensivo en el deporte. Sevilla: Wanceulen SL.

Cauas, D. (2015). Definición de las variables, enfoque y tipo de investigación. Bogotá, D.C.: Biblioteca electrónica de la universidad Nacional de Colombia.

Ceballos, P. (2010). Desde los ámbitos de enfermería, analizando el cuidado humanizado. Cienciayenfermería, 16(1), 31-35. http://dx.doi.org/10.4067/S0717-95532010000100004

Davini, M. C. (2008). Métodos de enseñanza. Didáctica general para maestros y profesores. Buenos Aires: Santillana.

Díaz, V. (2006). Formación docente, práctica pedagógica y saber pedagógico. Laurus, 12(Ext), 365-372. Recuperado de https://revistas.upel.edu.ve/index.php/sinopsis_educativa/article/viewFile/6130/3382

Díaz, P., Martínez, A. y Vernetta, M. (2004). Análisis de la metodología en la investigación sobre gimnasia rítmica. Lecturas: Educación física y deportes, 19(79). Disponible en https://www.efdeportes.com/efd79/ritm.htm

Fedecolgim. (2021). Gimnasia Artística Femenina. [Online]. Disponible en http://fedecolgim.co/gaf/gaf-definicion

Fernández-Río, J. (2013). Marco metodológico para la enseñanza de las habilidades gimnásticas en el ámbito educativo. Revista Española de Educación Física y Deportes, (400), 37. Disponible en https://www.reefd.es/index.php/reefd/article/view/140

Fernández-Río, J., Calderón, A., Alcalá, D. H., Pérez-Pueyo, Á. y Cebamanos, M. A. (2016). Modelos pedagógicos en educación física: consideraciones teórico-prácticas para docentes. Revista Española de Educación Física y Deportes, (413), 55-75. Disponible en https://www.reefd.es/index.php/reefd/article/view/425

Fraile, A. (2004). Hacia un deporte escolar educativo. En , A. Fraile (Coord.), El deporte escolar en el siglo XXI: análisis y debate desde una perspectiva europea (pp. 9-30). Barcelona: Graó.

Giglio, M. (2013). Cuando la colaboración creativa cambia la forma de enseñar: Desarrollo y observación de una propuesta pedagógica desde la educación musical. Santander: Universidad de Cantabria.

Giménez, F. J., Abad, M. T. y Robles, J. (2010). El proceso de formación del jugador durante la etapa de iniciación deportiva. Apunts. Educación Física y Deportes, (99), 47-55. Disponible en http://hdl.handle.net/10272/10200

Gómez, A. (2005). La enseñanza y el aprendizaje de los valores en la educación Deportiva. Revista Internacional de Medicina y Ciencias de la Actividad Fisica y el Deporte, 5(18), 89-99. Disponible en http://cdeporte.rediris.es/revista/revista18/arteduvalores9.htm 
Gómez-Mármol, A., Calderón-Luquin, A. y Valero-Valenzuela, A. (2014). Análisis comparativo de diferentes modelos de enseñanza para la iniciación al atletismo. Ágora para la Educación Física y el Deporte, 16(2), 104-121. Disponible en https://revistas.uva.es/ index.php/agora/index

González, S., García, L. M., Contreras, O. R. y Sánchez-Mora, D.. (2009). El concepto de iniciación deportiva en la actualidad. Retos. Nuevas tendencias en Educación Física, Deporte y Recreación, (15), 14-20. https://doi.org/10.47197/retos.v0i15.34992

González, H. (2015). La integración de la tecnología como herramienta significativa en la enseñanza del inglés como lengua extranjera. Revista Horizontes Pedagógicos, 17(1), 53-66. Disponible en https://horizontespedagogicos.ibero.edu.co/article/view/17105

González, H., Villota, J. y Riofrio, E. (2019). Modelos de aprendizajes virtuales y presenciales en lecto-escritura. Horizontes Pedagógicos, 21(1), 15-34. https://doi.org/10.33881/01238264.hop. 21102

González, S., Ibáñez, S. J., Feu, S. y Galatti, L. R. (2017). Programas de intervención para la enseñanza deportiva en el contexto escolar, PETB y PEAB: Estudio preliminar. Retos, 31, 107-113. https://doi.org/10.47197/retos.v0i31.43545

Guillen, L. G., Copello, M. C., Gutiérrez, M. G., y Guerra, J. (2018). Metodología para el perfeccionamiento del proceso de enseñanza-aprendizaje de los elementos técnicostácticos en los deportes de combate. Retos: nuevas tendencias en educación. Retos, 34, 33-39. https://doi.org/10.47197/retos.v0i34.58752

Hernández, B. y Velasco-Mondragón, H. E. (2000). Encuestas transversales. Salud pública de México, 42, 447-455. Disponible en https:/www.saludpublica.mx/index.php/ spm/article/view/6263

Hernández, E. (2015). El B-learning como estrategia metodológica para mejorar el proceso de enseñanza-aprendizaje de los estudiantes de inglés de la modalidad semipresencial del departamento especializado de idiomas de la Universidad Técnica de Ambato. [Doctoral dissertation]. Universidad complutense de Madrid, España. recuperado de https://core.ac.uk/download/pdf/33103212.pdf

López, P. L. (2004). Población muestra y muestreo. Punto cero, 9(08), 69-74. Disponible en https://cba.ucb.edu.bo/universidad/publicaciones/revistas-2/punto-cero/

Martínez, C. y Galán, A. (2014). Técnicas e instrumentos de recogida y análisis de datos. Madrid: Editorial UNED.

Morales, O. (2003). Fundamentos de la investigación documental y la monografía. Manual para la elaboración y presentación de la monografía. Mérida: Universidad de Los Andes.

Ortiz, A. y Canto, P. (2013). Estilos de aprendizaje y rendimiento académico en estudiantes de ingeniería en México. Revista de estilos de aprendizaje, 6(11), 160-177. Disponible en http://revistaestilosdeaprendizaje.com/article/view/978

Otero, A. (2018). Enfoques de Investigación. Métodos para el diseño del proyecto de Investigación. 
Pires, F. y Yanes, C. M. (2013). Diseño de estrategias metodológicas innovadoras en los procesos de enseñanza-aprendizaje y creación de espacios formativos en la asignatura teoría e historia de la educación física y el deporte. Revista de Enseñanza Universitaria, 39, 1-13. Disponible en http://hdl.handle.net/11441/52054

Rodríguez, H., Lara, D. y Rodríguez, C. E. (2018). El profesor de la iniciación deportiva y la evaluación de su desempeño. Lecturas: Educación Física y Deportes, 22(237), 64-69. Disponible en https://www.efdeportes.com/efdeportes/index.php/EFDeportes/ article/view/77

Sánchez, R. y Martín, J. (2019). ¿Más espectacular o más peligroso? Cambios recientes en el equilibrio de tensiones en gimnasia artística. Revista Española de Sociología, 28(3), 461-474. http://dx.doi.org/10.22325/fes/res.2019.11

Santos, L. (2020). Ámbitos de desarrollo de la Gimnasia Artística en el Uruguay. Lecturas: Educación Física y Deportes, 25(270), 83-93. https://doi.org/10.46642/efd.v25i270.2127

Toro, A. E., Álvarez, I. D. y Benjumea, M. M. (septiembre, 2013). Recréate con los otros: Una experiencia significativa para la convivencia escolar. Aporte desde la Educación Física, la recreación y el deporte. Presentado al $10^{\circ}$ Congreso Argentino y $5^{\circ}$ Latinoamericano de Educación Física y Ciencias, Universidad Nacional de La Plata, La Plata, Argentina. Recuperado de http://congresoeducacionfisica.fahce.unlp.edu.ar/10oca-y-5o-1-efyc/actas-10-y-5/Eje1_MesaG_Alvarez.pdf

Tous, E. E. (2002). La acrobacia en gimnasia artística: su técnica y su didáctica. Barcelona: INDE.

Valencia, H. G., Enríquez, J. A. V. \& Agredo, P. M. (2017). Strategies Used by Professors through Virtual Educational Platforms in Face-To-Face Classes: A View from the Chamilo Platform. English Language Teaching, 10(8), 1-10. https://doi.org/10.5539/elt. v10n8p1

Vargas, E. (2020). Modelos de colaboración docente en red para el área de música. Un análisis de los proyectos eTwinning en el período 2008-2018. [Tesis doctoral]. Universidad de Castilla-La Mancha, Toledo, España. Disponible en http://hdl.handle. net/10578/27242

Heriberto González Valencia es Doctorando en Investigación en Humanidades Artes y Educación de la Universidad Castilla La Mancha (España). Magister en Educación, Especialista en Educación y Licenciado en Lenguas Extranjeras. Miembro activo del grupo de investigación EDUCAR 2030 de la Institución Universitaria Escuela Nacional del Deporte (Cali, Colombia). https://orcid.org/0000-0001-9103-2152

German Darío Isaza Gómez es Doctorando en Educación de la Universidad de la Salle (Costa Rica). Magister en Educación. Director del grupo de investigación EDUCAR 2030 de la Institución Universitaria Escuela Nacional del Deporte (Cali, Colombia). https://orcid. org/0000-0001-8475-9994

Luz Karime Parra Estrada es profesional en Deportes de la Institución Universitaria Escuela Nacional del Deporte. Cali (Colombia). https://orcid.org/0000-0003-4184-9521 